\title{
Poison Control Center Data in the NC DETECT Syndromic Surveillance System
}

\author{
Zachary Faigen $^{\star 1}$, Lana Deyneka ${ }^{1}$, Anne Hakenewerth ${ }^{1}$ and Michael C. Beuhler ${ }^{2}$ \\ ${ }^{1}$ North Carolina Department of Health and Human Services, Raleigh, NC, USA; ${ }^{2}$ Carolinas Poison Center, Charlotte, NC, USA
}

\section{Objective}

To describe Carolinas Poison Control Center (CPC) calls data collected in the NC DETECT syndromic surveillance system.

\section{Introduction}

CPC provides the $24 / 7 / 365$ poison hotline for the entire state of North Carolina and currently handles approximately 80,000 calls per year. CPC consultation services that assist callers with poison exposure, diagnosis, optimal patient management, therapy, and patient disposition guidance remain indispensable to the public and health care providers. Poison control center data have been used for years in syndromic surveillance practice as a reliable data source for early event detection. This information has been useful for a variety of public health issues, including environmental exposures, foodborne diseases, overdoses, medication errors, drug identification, drug abuse trends and other information needs. The North Carolina Department of Health and Human Services started formal integration of CPC information into surveillance activities in 2004. CPC call data are uploaded in real time (hourly), 24/7/365, to the NC DETECT state database.

\section{Methods}

CPC calls collected by NC DETECT from 2009-2015 were analyzed in this descriptive study. Counts of CPC calls were examined by year to assess total volume and changes over time, by month to assess seasonality, by geographic location, and call site facility and call originator. CPC calls were also categorized by type of call - exposure calls versus information calls - in order to determine why people call CPC and to assess if any trends exist amongst these categories.

\section{Results}

The majority of CPC calls originate from the caller's own residence $(53.40 \%)$. The age groups most represented are $0-1$ years old, 2-4 years old, and 25-44 years old. Calls to CPC were for male and female patients in approximately equal numbers. The region of $\mathrm{NC}$ that has the highest number of calls, by a fairly wide margin, is the Charlotte Metro region. In 2009, the total number of CPC calls was over 120,000 . This number decreased monotonically every year following, with the total in 2015 being 80,000 . This is a $1 / 3$ reduction in the total number of calls over 7 years. When the calls were analyzed by type of call, an interesting trend emerged. The total number of exposure calls remained relatively constant over the time period, ranging from 64,000 to 68,000 per year. However, the total number of information calls decreased each year going from just over 40,000 to only about 5,000 . When examined by month to assess seasonality, the data show an increase in the number of calls beginning in February and peaking in May, and then a steady and slow decline throughout the rest of the year.

\section{Conclusions}

Our study shows that CPC consultations from callers with exposures have remained stable over time. However, in the absence of exposure, fewer people call CPC for information on various substances. Drug identification calls saw a decrease each year during the study time period. In 2009 there were 34,495 drug identification calls and in 2015 there were 5,722. This dramatic decrease in information calls is most likely due to the increased use of the internet and search engines. Because people have more access to the internet, especially via mobile devices, they may not feel the need to call CPC to obtain information.

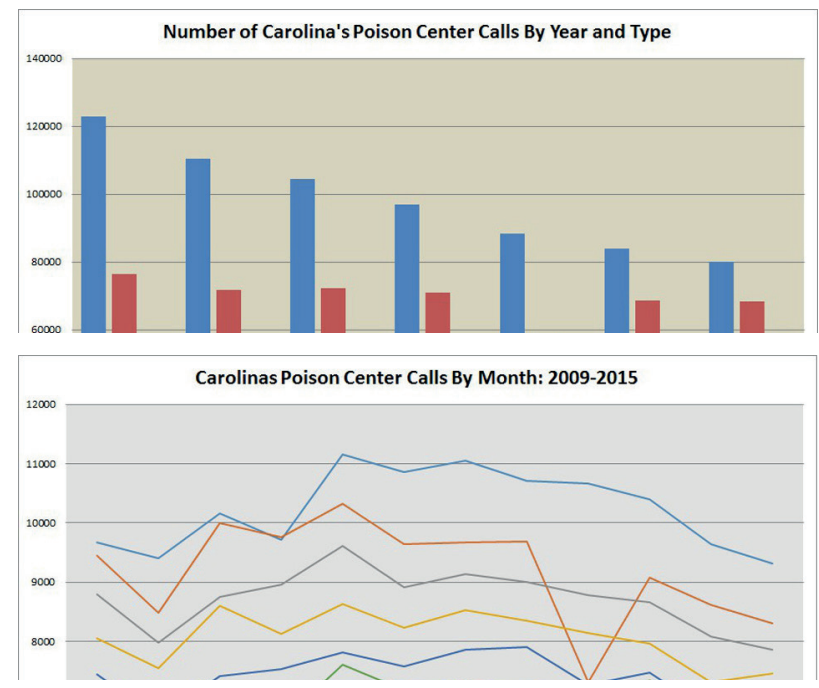

\section{Keywords}

syndromic surveillance; poison control center; descriptive study; data evaluation

\section{Acknowledgments}

We would like to acknowledge all those involved with the NC DETECT program at both the North Carolian Department of Health and Human Services and The University of North Carolina at Chapel Hill, as well as the Carolinas Poison Center.

\section{*Zachary Faigen}

E-mail: zachary.faigen@dhss.nc.gov 